\title{
A versatile maze for learning and geotaxic selection in Drosophila melanogaster
}

\author{
OWEN W. DRUDGE and STEVE ANDERSON PLATT \\ Northern Michigan University, Marquette, Michigan 49855
}

\begin{abstract}
Behavior geneticists have long sought to display learning in the fruit fly (Drosophila melanogaster), but previous research efforts have proven problematic. The following describes a maze apparatus and a procedural technique that have produced positive results in learning tasks with negative geotaxis flies. The Plexiglas maze is inexpensive to construct and offers a functional versatility that makes it adaptable to many different experimental applications.
\end{abstract}

The fruit fly (Drosophila melanogaster), noted for its rapid rate of reproduction and minimal maintenance requirements, has been the perennial subject of genetic research. The resultant accumulated knowledge of its chromosomal structure has made it a natural choice for investigating the genetic components of learning. Historically, demonstrating such learning in Drosophila has proven problematic at best. Lack of successful replications (McGuire \& Hirsch, 1977; Yeatman \& Hirsch, 1971) and alternative explanations of data (Bicker \& Spatz, 1976; Murphey, 1973) have been predominant in previous efforts. The following successful methodology evolved as an attempt to circumvent these shortcomings.

\section{METHOD}

Murphey (1967), utilizing Hirsch strain negative geotaxic fruit flies (Hirsch, 1963), reportedly demonstrated instrumental conditioning by pairing right or left $\mathrm{T}$-maze choices with an opportunity to ambulate upward through a vertical arm of a tubular maze. He hypothesized that the selectively bred geotaxic behavior (i.e., the gravity-oriented locomotion) would act as a reinforcer and would facilitate learning over trials. Subsequent efforts to replicate this approach have failed (Yeatman \& Hirsch, 1971), leading to a reinterpretation of these data and a retraction of any earlier claims of conditioning (Murphey, 1973). Murphey's T-maze design employed single-choice trials after which the subjects were transferred to the start tube for the following trial repetitions. This disruptive and repeated handling could have competed with the attentional factors necessary for learning in that situation. It is therefore possible that the original Murphey hypothesis was correct, but that his apparatus and experimental technique were incapable of demonstrating it. It was from these observations that the following maze design emerged.

\section{Apparatus}

The Plexiglas maze design was developed with emphasis on

Requests for reprints should be addressed to Owen W. Drudge, Department of Psychology, John Dewey Hall, University of Vermont, Burlington, Vermont 05401. The authors wish to offer special thanks to Dr. Jerry Hirsch for furmishing the positive and negative geotaxic strains for research purposes. maximizing versatility and minimizing costs. Adopting geotaxic behaviors as a means of reinforcement in learning experimentation necessitates continual geotaxic selection to maintain the quality of the strain and the salience of the trait. The described maze can be used for studying T-maze learning and Hirsch-type geotaxic selection.

Chassis construction consists of cementing $1.5 \times 4 \mathrm{~cm}$ rectangular sections of $.25-\mathrm{in}$. $(.64-\mathrm{cm})$ clear Plexiglas onto a .25-in. $(.64-\mathrm{cm})$ Plexiglas base sheet. This produces a matrix (Figure 1) with $.25-\mathrm{in} .(.64-\mathrm{cm})$ spacings between the 16 rows and 18 columns, forming a series of square alleys throughout the maze. Various pathways are established by inserting .25 -in $(.64-\mathrm{cm})$ rubber plugs to block off appropriate regions. The unit is completed with a Plexiglas cover that is attached by bolts to the chassis and a black plywood backing for structural support.

The left lateral portion of the maze is utilized for the purpose of geotaxic selection, as shown in Figure 1. The systematic placement of rubber plugs creates a seven-choice eight-unit maze with $1.5-\mathrm{cm}$ horizontal alleys leading to $8.64-\mathrm{cm}$ ascending and descending vertical arms or alleys. As is common with almost all selection mazes of this sort, choice points are isolated by cones to prevent the flies either from reversing their paths (i.e., backtracking through the maze) or from progressing to the adjoining unit (Figure 2). These cones are constructed by machining conical openings into $.25-\mathrm{in} .(.64-\mathrm{cm})$ square by $1-\mathrm{cm}$ rectangular sections of Plexiglas and are hand press-fitted into their designated locations. Eight plastic Drosophila vials (Carolina Biological Supply Company) are attached to the maze cover plate to serve as collection tubes, as shown in Figure 3.

The opposing right lateral portion of the maze serves as a seven-choice vertical $T$-maze for learning experimentation. Correct placement of rubber plugs produces a series of $8.64-\mathrm{cm}$ vertical pathways, culminating in " $\mathrm{T}$ " choice points with $1.5-\mathrm{cm}$ horizontal arms stemming in each direction. One arm terminates in a cul-de-sac, while the other allows the animal to proceed upward to the next choice point (Figure 1). A wall-hugging tendency (i.e., ambulation on one specific surface of the maze alley), which strongly biases responses at choice points, has been noted by other researchers; it was also observed in pilot studies with this maze prototype. Wall hugging was eliminated by adding .6-cm-long cones at each of the choice points. In comparison with the geotaxic cones, these are considerably shorter and have larger exit openings; this serves to allow reasonably unbiased choice responding while interfering minimally with the flies' upward progress. Unlike more traditional experimental learning animals, flies possess the uncanny ability to walk on all surfaces of the maze. Simple right-left choices consequently offer less kinesthetic information about the maze layout (i.e., the fly's left choice on the back maze surface is in the same 


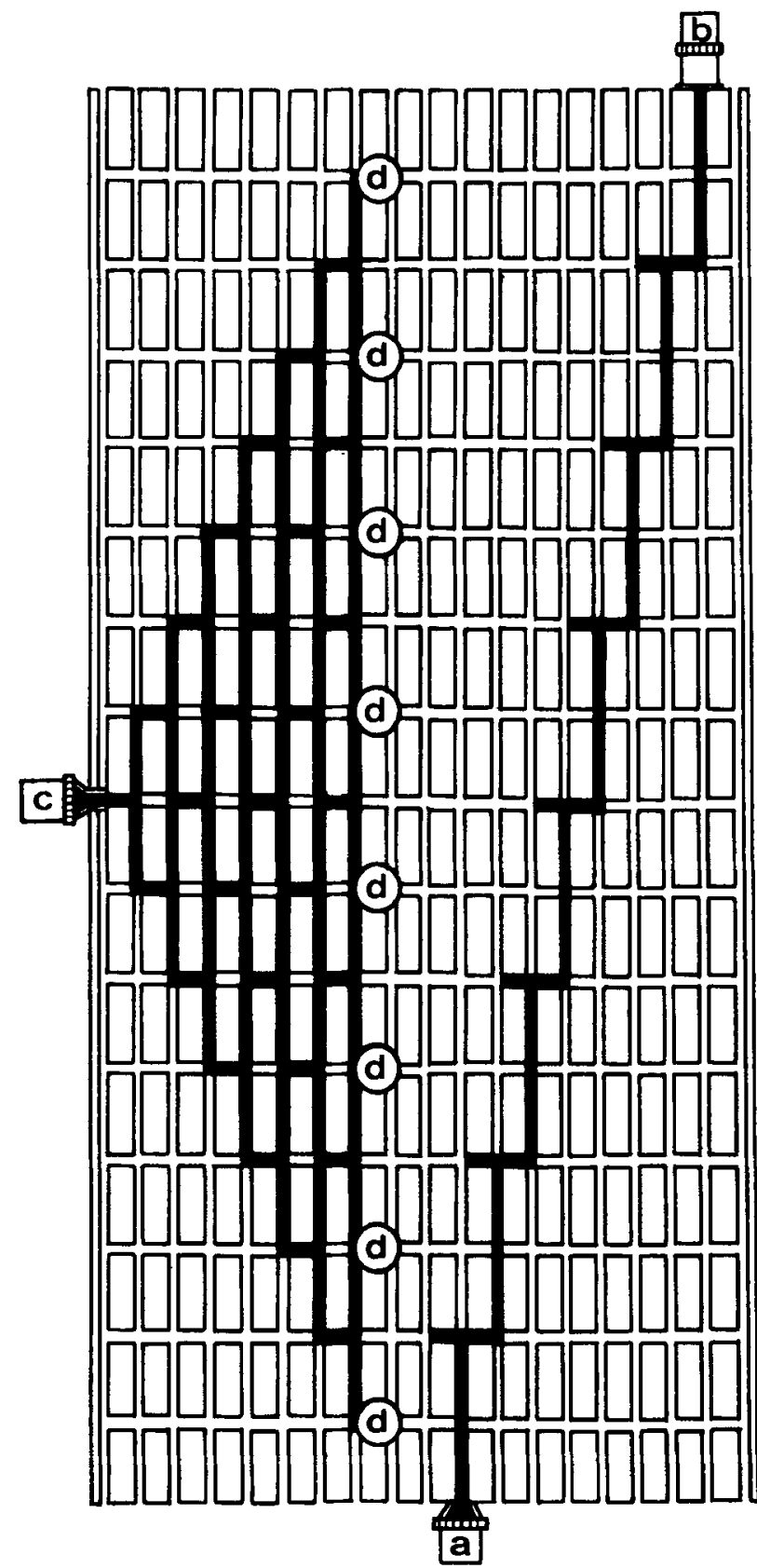

Figure 1. Schematic layout of basic maze design with geotaxic selection portion on left and one of the many possible learning configurations on right. The wide black lines denote pathways through maze. Also shown are (a) learning start tube, (b) learning collection tube, (c) geotaxic start tube, and (d) geotaxic collection tubes.

direction as the fly's right choice on the front). To increase the available discrimination cues, sandpaper strips $1.8 \mathrm{~cm}$ long are placed on one side of the choice point, covering the top, back, and bottom of the alley (Figure 4).

In pilot studies, we observed that any diffuse light coming through the maze and unilaterally illuminating one side of the horizontal alley more than the other influenced choice point behavior. The positive phototropic or prepotent tendency to
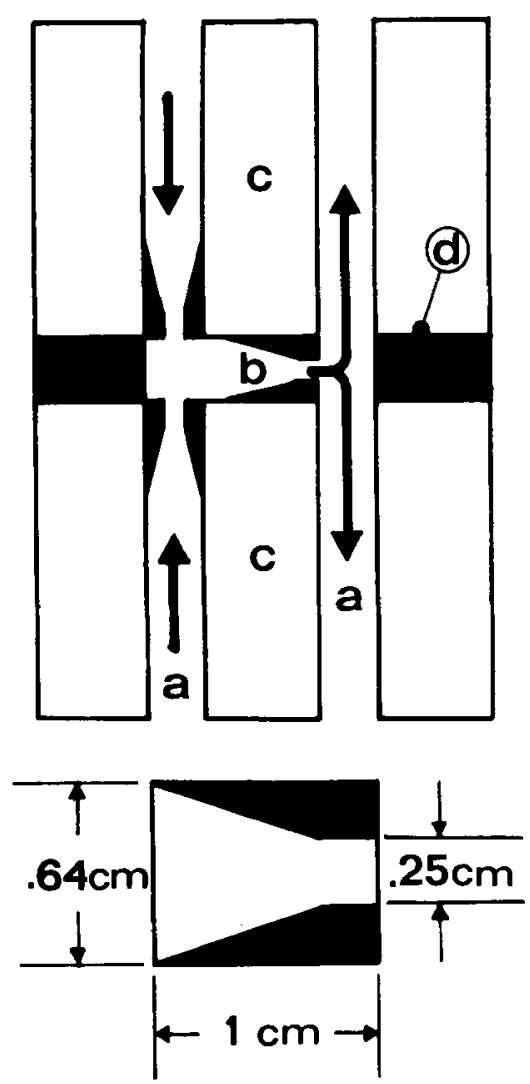

Figure 2. Enlanged section of geotaxic selection maze showing (a) pathways, (b) cone, (c) Plexiglas rectangular sections, and (d) rubber plug.

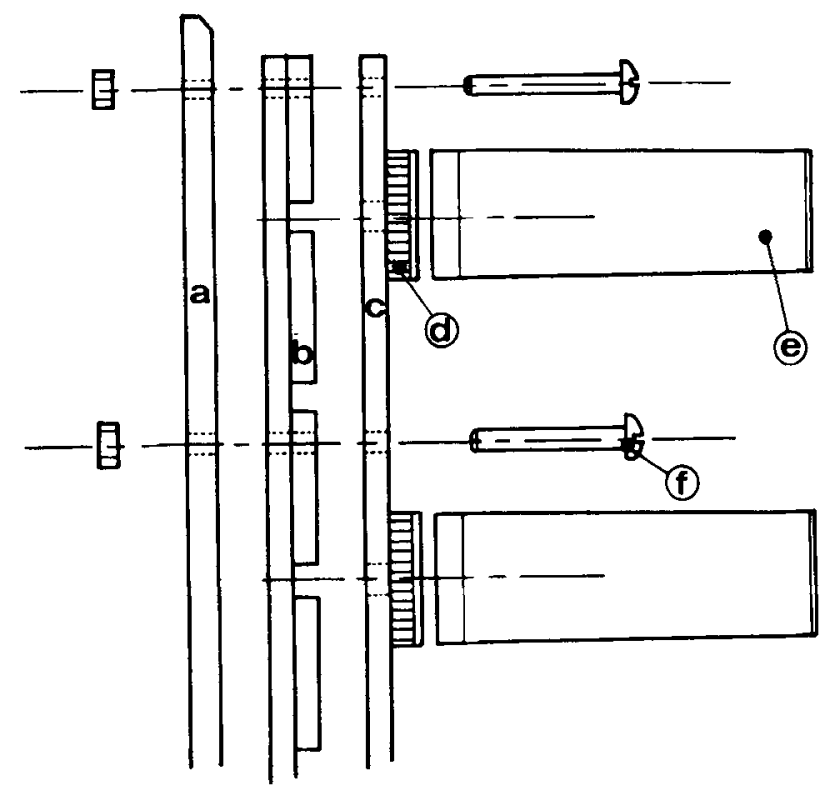

Figure 3. Side view of maze assembly with (a) plywood backing, (b) maze chassis, (c) cover plate, (d) vial cap, (e) plastic Drosophila vial, and (f) $1.25 \times .25$ in. bolt. 
approach light sources, in such cases, interferes with other maze behaviors. To alleviate this problem, .25-in. $(.64-\mathrm{cm})$ aluminum foil tape is adhered along the upper surface of the horizontal arms. For proper counterbalancing and controls, the rubber plugs, cones, and sandpaper strips can easily be rearranged to establish the desired configuration. Start and collection tubes are interchangeable and are fashioned from plastic Drosophila vials.

\section{Procedure}

The procedure for geotaxic selection is relatively simple. The maze is placed in a vertical upright position with a $20-\mathrm{W}$ fluorescent light placed directly in front of and parallel to the maze. Flies, in groups of up to 100 , are slowly fed in through the start tube situated on the lateral edge of the maze chassis (Figure 1). After a short period of time, 2-20 min, the flies emerge in any of the eight collection tubes protruding from the cover midline. They are assigned geotaxic scores from 0 to 7 , depending on the number of "upward" choices the animal makes while passing through the maze.

For use in learning tasks, the maze is stationed in the same vertical position with a 15 -W fluorescent light located approximately $20 \mathrm{~cm}$ above the collection tube to encourage rapid passage through trials. Single negative geotaxic $D$. melanogaster (those that ambulate away from gravity) are placed into the start tube at the bottom of the maze. As the fly proceeds through the maze, its behavior is noted at each choice point; correct vs. incorrect choices are tabulated. A performance score is assigned according to the fly's number of correct responses; this can range from 0 to 7 (one possible per choice point). This is repeated by isolating the animal in the lower half of
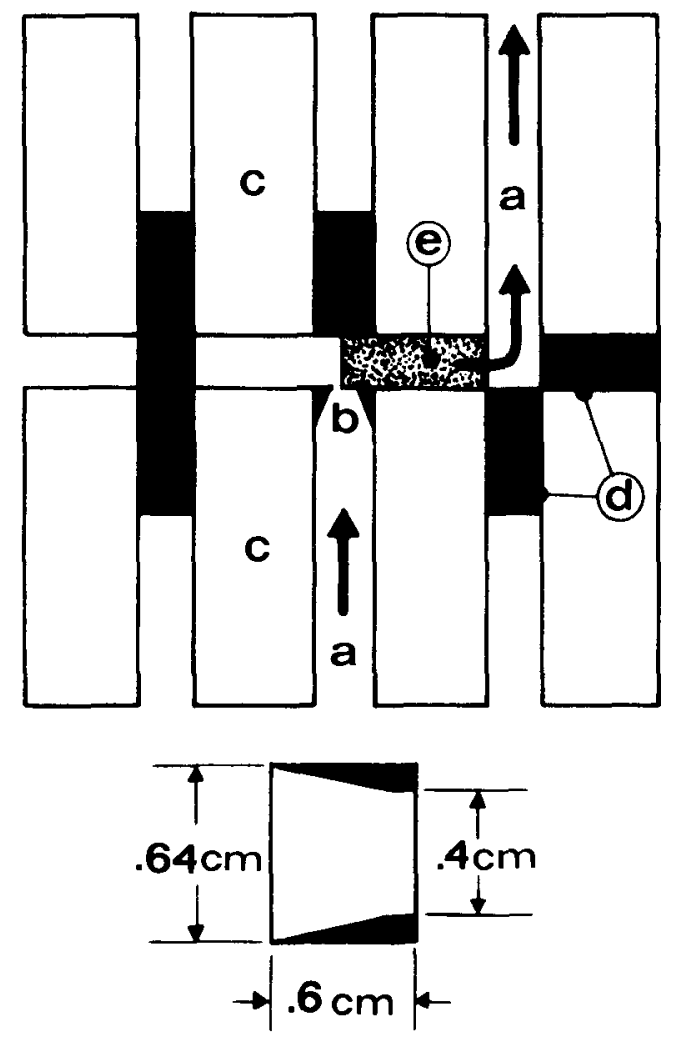

Figure 4. Enlarged section of learning maze showing (a) pathways, (b) cone, (c) Plexiglas rectangular sections, (d) rubber plugs, and (e) sandpaper strip.

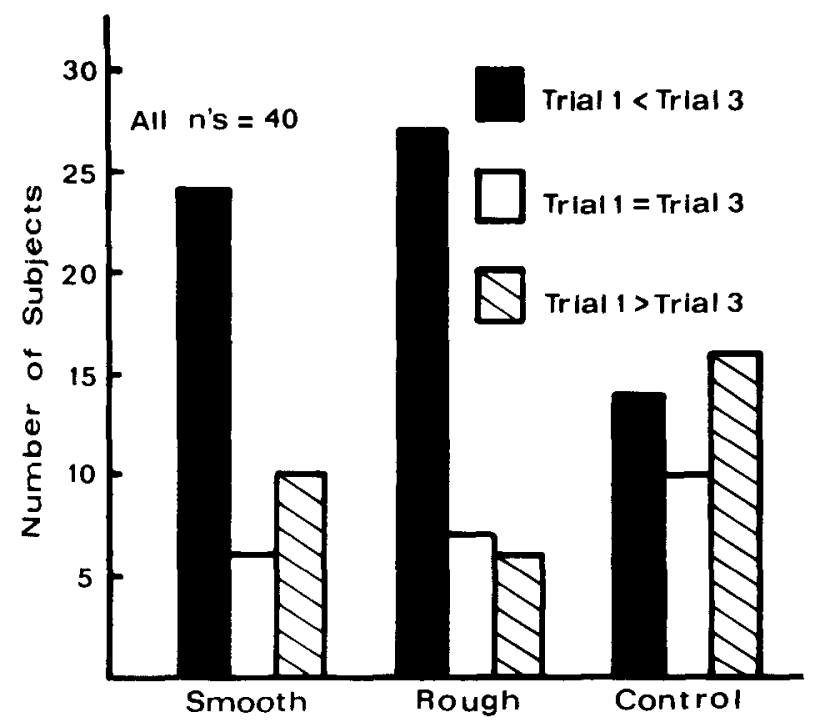

Figure 5. Shown is the comparison of the number of correct responses by subjects on first passage through maze (Trial 1) with the last passage (Trial 3). Key: Black bar denotes those subjects improving score; white bar denotes those showing no score change; slashed bar denotes those achieving lower score on last trial. Experimental groups, smooth (away from sandpaper) and rough (over sandpaper), showed a significantly large number of animals increasing scores, while the control group displayed no indication of learning.

the two-section collection tube, which is transferred to become the start tube for the following trial. Repeated trials are scored and compared with earlier scores to evaluate possible increases in performance: learning.

\section{DISCUSSION}

In a recent learning study (Platt \& Drudge, Note 1), counterbalanced for gender, choice direction, and stimulus substrate (over vs. away from sandpaper), Hirsch-strain negative geotaxic flies showed consistent significant score improvement over trials. A control group given inconsistent cues, alternating right-left and sandpaper/no-sandpaper choices, showed no increase in correct responses (Figure 5). The lack of improvement by the control subjects tends to discount competing explanations (e.g., pheromonal odor trails). The availability of such nonlearning-induced score improvements was equivalent for both the experimental and control animals, the only essential difference being the presence or absence of relevant, systematic cue stimuli.

By simply rotating the structure $180 \mathrm{deg}$, the previously described technique can be utilized with positive geotaxic flies (those that ambulate toward gravity). Manipulating the placement of cones and rubber plugs yields countless possible run patterns or configurations. It is this exceptional flexibility of the maze that makes it a valuable instrument for both classroom demonstrations and advanced research in the fields of behavior genetics and insect learning. 


\section{REFERENCE NOTE}

1. Platt, S. A., \& Drudge, O. W. Maze learning in Drosophila melanogaster. Poster presentation at the annual meeting of the American Association for the Advancement of Science, Houston, January 1979. Manuscript available from authors. (Abstract 625).

\section{REFERENCES}

Bicker, G., \& Spatz, H. Сh. Maze-learning ability of Drosophila melanogaster. Nature, 1976, 260, 371.

HiRsch, J. Behavior genetics and individuality understood. Science, 1963, 142, 1436-1442.

McGuire, T. R., \& Hirsch, J. Behavior-genetic analysis of
Phormia regina: Conditioning, reliable individual differences, and selection. Proceedings of the National Academy of Sciences, 1977, 74, 5193-5197.

Murphey, R. M. Instrumental conditioning of the fruit fly, Drosophila melanogaster. Animal Behaviour, 1967, 15, 153-161.

Murphey, R. M. Spatial discrimination performance of Drosophila melanogaster: Test-retest assessments and a reinterpretation. Animal Behaviour, 1973, 21, 687-690.

Yeatman, F, R., \& Hirsch, J. Attempted replication of, and selective breeding for, instrumental conditioning of Drosophila melanogaster. Animal Behaviour, 1971, 19, 454-462.

(Received for publication January 31, 1979; revision accepted June $26,1979$. ) 